\title{
Investigation of visual space using an exocentric pointing task
}

\author{
R. H. CUIJPERS, A. M. L. KAPPERS, and J. J. KOENDERINK \\ Helmholz Instituut, Utrecht, The Netherlands
}

\begin{abstract}
Classically, it has been assumed that visual space can be represented by a metric. This means that the distance between points and the angle between lines can be uniquely defined. However, this assumption has never been tested. Also, measurements outdoors, where monocular cues are abundant, conflict with this model. This paper reports on two experiments in which the structure of visual space was investigated, using an exocentric pointing task. In the first experiment, we measured the influence of the separation between pointer and target and of the orientation of the stimuli with respect to the observer. This was done both monocularly and binocularly. It was found that the deviation of the pointer settings depended linearly on the orientation, indicating that visual space is anisotropic. The deviations for configurations that were symmetrical in the median plane were approximately the same, indicating that left/right symmetry was maintained. The results for monocular and binocular conditions were very different, which indicates that stereopsis was an important cue. In both conditions, there were large deviations from the veridical. In the second experiment, the relative distance of the pointer and the target with respect to the observer was varied in both the monocular and the binocular conditions. The relative distance turned out to be the main parameter for the ranges used $(1-5 \mathrm{~m})$. Any distance function must have an expanding and a compressing part in order to describe the data. In the binocular case, the results were much more consistent than in the monocular case and had a smaller standard deviation. Nevertheless, the systematic mispointings remained large. It can therefore be concluded that stereopsis improves space perception but does not improve veridicality.
\end{abstract}

Most of the time, people are unaware of any discrepancies between the visually perceived environment and the physical environment. However, early experiments on size constancy (e.g., Blumenfeld, 1913; Hillebrand, 1902) revealed that the visually perceived space (in short, visual space) is distorted with respect to the physical space. In order to explain these findings, Luneburg (1947) proposed a theoretical framework in which he assumed that, in nature, visual space is a constantly curved Riemannian space. For example, the surface of a sphere is a twodimensional Riemannian space with a constant positive curvature, and a surface with a constant negative curvature is saddle shaped (see any book on differential geometry, such as Stoker, 1969). After that, the focus of research shifted and was directed toward determining the curvature of visual space (Blank 1958, 1961; Hardy, Rand, \& Rittler, 1951; Indow, 1991; Indow, Inoue, \& Matsushima, 1962a, 1962b; Indow \& Watanabe, 1984; Zajaczkowska, 1956a, 1956b). These authors described experiments carried out in a dark room with small faint luminous points as stimuli at distances ranging from 0.50 up to $5.00 \mathrm{~m}$. From the results, the curvature constant was then determined. During these experiments, the heads of the subjects were fixed, whereas free eye movement was

Correspondence concerning this article should be addressed to $\mathrm{R}$. $\mathrm{H}$. Cuijpers, Helmholtz Instituut, Princetonplein 5, 3584 CC Utrecht, The Netherlands (e-mail: a.m.I.kappers@phys.uu.nl). allowed. This reduced-cue environment, in which most of the monocular cues were eliminated, actually closely approached the assumptions made in Luneburg's theory. But in everyday life, we have a full-cue environment and broad daylight, which is very different from the dark room experiments. More recently, similar experiments have been carried out in the open air in order to find out to what extent visual space is distorted under these conditions. For example, Battro and his colleagues (Battro, di Pierro Netto, \& Rozestraten, 1976; Battro, Reggini, \& Karts, 1978) performed their studies in broad daylight in gardens and on polo fields over distances up to $240 \mathrm{~m}$. Wagner (1985) took measurements on a flat open grassy field over distances up to $72 \mathrm{~m}$. These studies show that Luneburg's model is less adequate in a full-cue environment, in which monocular cues are abundant, and that stereopsis is less effective because of the large distances. Apparently, the structure of visual space depends on the actual environment in which it is measured, and the initial assumptions about the metrical structure of visual space need to be examined more closely.

Luneburg (1947) assumed, for instance, that visual space can be represented by one of the Riemannian spaces of constant curvature. He also assumed that lights positioned on a Vieth-Müller circle are perceived as lying on a circle with the observer at its center. Both assumptions have been shown to be, at least partly, incorrect (Foley, 1963, 1965, 1972; Heller, 1997b; Higashiyama, 1981, 1984). Discussions about the theoretical consequences 
for models of visual space may also be found in Blank (1978), Eschenburg (1980), Heller (1997a), Indow (1991, 1997), and Lukas (1983).

A more fundamental assumption is that visual space is metric. This means that a unique distance can be defined between any two points. In order to investigate this, a task is needed that does not require the existence of a distance relation.

In the experiments reported in this paper, we used an exocentric pointing task for our measurements. In such a task, the subject is asked to direct a pointer straight at a target. The pointer, however, is placed at some distance from the subject and is operated by remote control. As a consequence, the pointer does not lie in the line of sight, as would normally be the case (e.g., aiming a rifle), but is placed at a distance from the egocenter. In principle, the pointer could be placed at any point in the surrounding space, but for the purpose of this paper, the pointer was always placed at eye height. Although there are only a few references to pointing tasks in the literature (e.g., Ellis, Smith, Grunwald, \& McGreevy, 1991), a pointing task turns out to be successful in obtaining properties of visual space, as was shown by Koenderink and van Doorn (1998). Furthermore, the task comes very naturally to subjects: It resembles a common practice in daily life in which a person follows the gaze of another person in order to find out what he or she is looking at.

In the exocentric pointing task, the subject needs to estimate directions that may, in principle, be estimated solely on the basis of a single retinal image without making use of depth cues. However, in order to point veridically, knowledge about the distance of the pointer relative to the target is required. Therefore, we will assume that the intrinsic geometry of visual space will be reflected in this exocentric pointing task as in the classical experiments (Indow, 1991), even though estimating directions may be an entirely different process.

In many studies, visual space is measured in the binocular condition only, while it is tacitly assumed that stereopsis is the only relevant cue. In the classical dark room experiments, this is bound to be true, since there is nothing visible except small faint luminous points with no reference whatsoever. Out of doors this is less obvious, because then there are numerous monocular cues present. Is stereopsis the main factor in estimating distances in realistic settings, or are monocular cues equally important? In order to verify that stereopsis is the relevant cue in the binocular condition, we also measured in the monocular condition. The influence of stereopsis will only be large if it provides a considerable amount of additional information. For this reason, we reduced the number of monocular cues by keeping the visual angle of the stimuli constant and by concealing external reference lines as much as possible without dimming the lights.

In a natural environment, people often turn their heads to direct their gaze to places of interest. In classical ex- periments, the heads of subjects were fixated, and only eye movements were allowed. In the outdoor experiments (Battro et al., 1976; Battro et al., 1978; Koenderink \& van Doorn, 1998; Wagner, 1985), subjects were also allowed to turn their heads. Free head movements will probably have a large influence on the symmetry of visual space. Under these circumstances, it will always be possible to fixate any point symmetrically with respect to the eyes. But will this possibility lead to an isotropic visual space? In our study, subjects were allowed to turn their heads. In order to address the symmetry properties, we varied the relative orientation of the stimuli with respect to the observer.

It was found earlier (Battro et al., 1978; Wagner, 1985) that a description of visual space in terms of a constantly curved space is less adequate under full-cue conditions. But it is still unclear whether visual space can be described adequately with a metric or, alternatively, with a single distance function. Which description is more appropriate and under what circumstances? In the present paper, we obtained the depth structure of visual space by measuring at different distances from the observer, both monocularly and binocularly. We will try to determine what constraints there are on any distance function and whether or not such a function is independent of the orientation of a stimulus configuration with respect to the subject. In addition, the influence of stereopsis can be accessed by comparing the viewing conditions.

In this paper, two experiments are presented in which the aforementioned questions were examined. In the first experiment, the monocular and binocular condition were compared when the stimuli were at the same distance from the observer. The dependence of the orientation of stimulus configurations with respect to the observer was investigated as well. The aim was to determine the role of stereopsis and to address the symmetries of visual space. In the second experiment, the relative distance of the stimuli was varied and measured both monocularly and binocularly. This should enable us to determine the constraints on a possible distance function. The role of stereopsis was addressed in this experiment as well.

\section{GENERAL METHOD}

\section{Subjects}

The measurements were carried out with 3 naive subjects, who were unfamiliar with the objectives of the experiments and had never seen the experimental environment before. Their age was approximately 22 years. All the subjects had normal or corrected-tonormal visual acuity. All the subjects were tested for stereo vision and were found to have a stereo acuity of better than 60 arcseconds. During all the measurements, the subjects had no feedback about their performance.

\section{Experimental Setup}

All the experiments were conducted in a $6 \times 6 \mathrm{~m}$ room with blinded windows under normal room-lighting conditions. The walls were covered with black plastic sheet material so that the corners of 
A

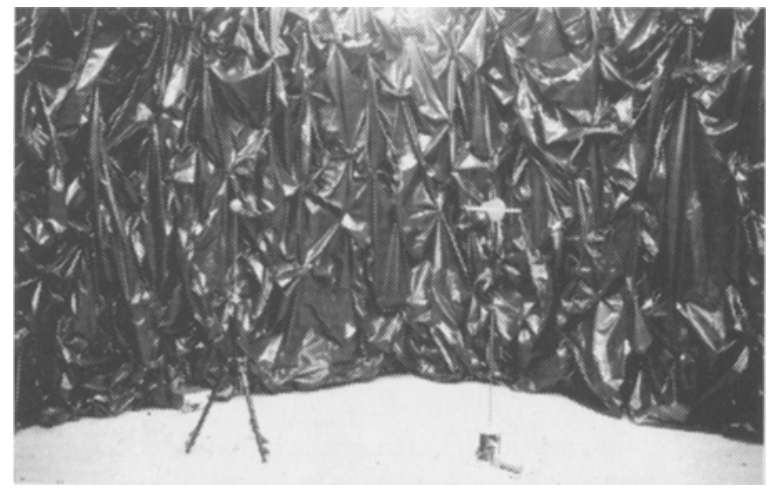

C

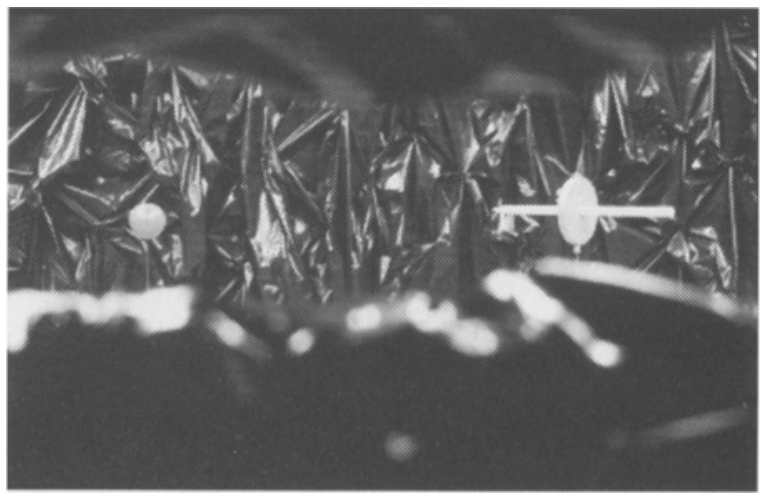

B

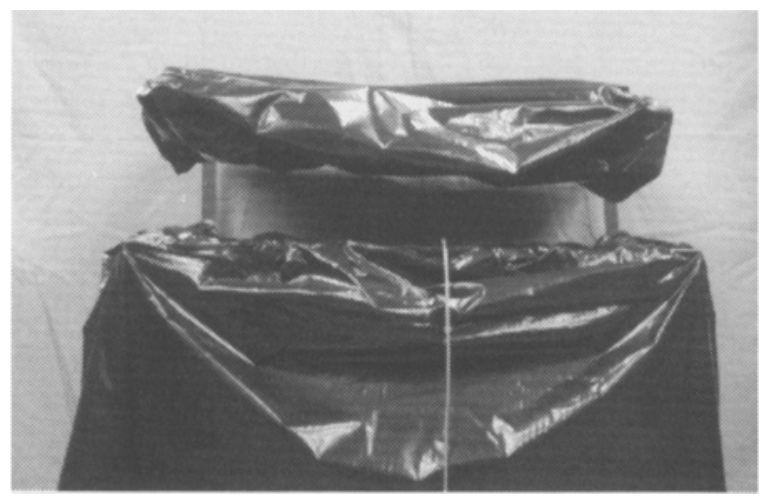

D

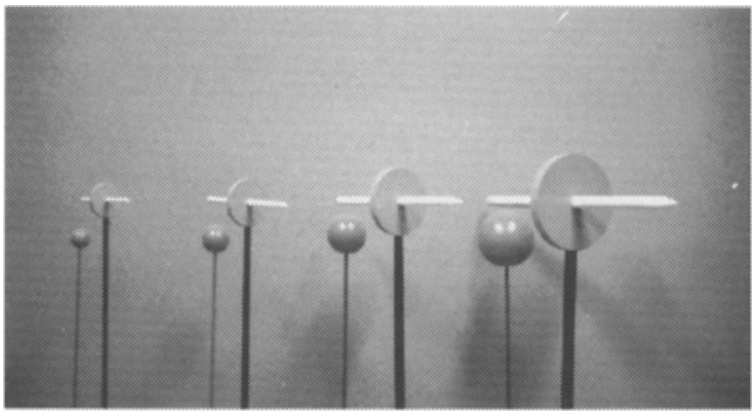

Figure 1. (A) Picture of the pointer and the target in the room in which the experiments were conducted. The heavy relief of the plastic that covers the walls is clearly visible. The pointer was mounted on a motor that was operated by remote control, and the target was placed on a tripod. (B) Picture of the outside of the front of the cabin in which the subject was seated. For clarity, the background of the photograph is gray; in the actual setting, the background consisted of black plastic. The cabin was $80 \mathrm{~cm}$ wide, and the space between the roof and the front was about $10 \mathrm{~cm}$. As a consequence, the subject was unable to see the floor and the ceiling of the room. The wire connected the remote control with the pointer. $(C)$ View from inside the cabin where only the pointer and the target remained visible against a "random" background. The total width of the visual field was much larger $\left(210^{\circ}\right)$ than depicted. (D) The various pointers and targets that were used during the experiments. Both were scaled with distance so that the apparent size remained constant.

the room were hidden and the general appearance of the background was the same in all directions. The plastic was wrinkled in order to create a heavy "random" relief: The extent of the protrusions and cavities was of the order of $10 \mathrm{~cm}$ (see Figure 1A).

The subject was seated on a chair, which was adjustable in height and which was kept in the same position with the same orientation. Since the subject was asked to sit up straight, the subject's body also was in the same position and orientation. The chair was placed inside a small cabin with a horizontal opening at eye height so that both the floor and the ceiling were hidden from view (see Figure $1 B$ ). The back of the cabin was open to allow easy access. Inside the cabin, a wooden board was mounted with a U-shaped hole in which the neck of a subject fitted. This simple construction prevented lateral head movements, but the subjects could still turn their heads and move their eyes freely. As a result, the entire visual field extended about $10^{\circ}$ vertically and $210^{\circ}$ horizontally (see Figure $1 \mathrm{C}$ ).

\section{Stimuli}

Four pointers and four targets of different sizes were used as stimuli (see Figure 1D and Table 1). Each target consisted of an or- ange sphere mounted on a thin metal rod standing upright on a tripod. Each pointer consisted of a rod with a pointed tip (top angle, $30^{\circ}$ ), which protruded on both sides at right angles from a circular disk. The rod was painted white, and the disk yellow-orange. The pointer was mounted on a thin metal rod, which was connected to a motor so that it could rotate in a horizontal plane. The motor was operated by remote control so that the pointer could be turned to the left or the right by pressing a switch. The height was adjusted so that both the pointer and the target were in a horizontal plane at eye height. Hence, only the pointer and the target itself were visible from within the cabin (Figure $1 \mathrm{C}$ ).

Both the pointer and the target were positioned using markers on the floor. The pointer-target positions were selected from the intersections of four lines of constant radius $(1.47,2.1,3.0$, and $4.31 \mathrm{~m}$ ), centered on the subject, with 15 lines of constant angle, ranging from $-105^{\circ}$ to $105^{\circ}$ in steps of $15^{\circ}$, emanating from the position of the subject (see Figure 2A). Henceforth, we will use $r_{\mathrm{p}}$ and $\psi$ as the polar distance and angle of the pointer position and $r_{\mathrm{t}}$ and $\theta$ as the polar coordinates of the target position (see Figure 2B). The indicated direction of the pointer is expressed with the absolute 
A

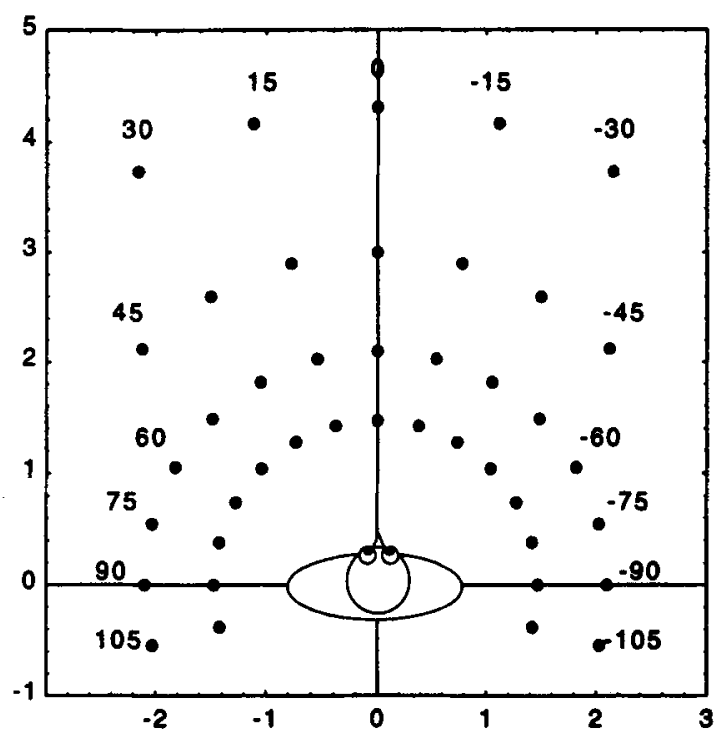

B

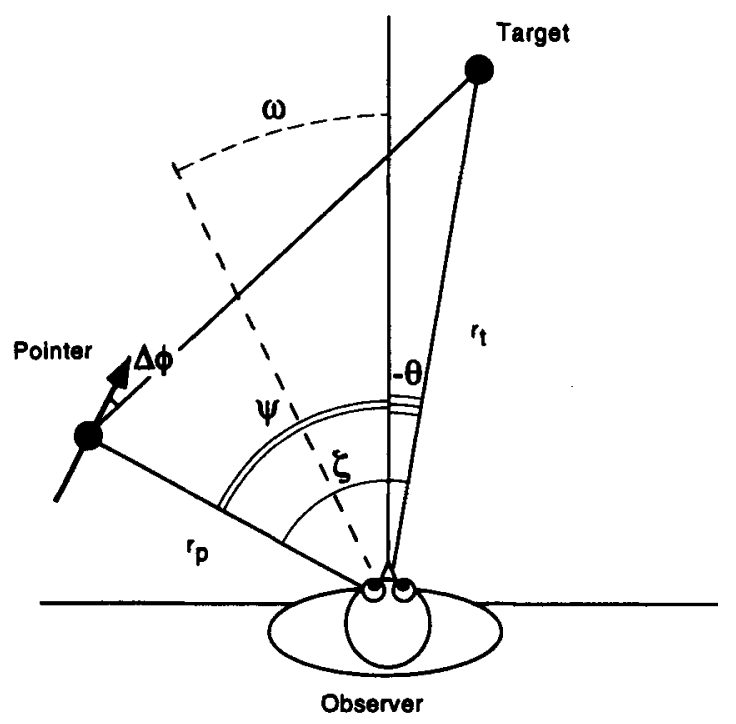

Figure 2. (A) Schematic diagram of the room in which the experiments were conducted. The subject was seated on a chair so that the turning point of the head was above the origin. The subject was oriented to face the $0^{\circ}$ direction when looking straight ahead. The dots denote the pointer and target positions that were used. (B) Diagram of the parameters used. The pointer and target positions are denoted by the distance from the origin, $r_{\mathrm{p}}$ and $r_{\mathrm{t}}$, and by the polar angles $\psi$ and $\theta$. The deviation of the indicated angle from the veridical is denoted by $\Delta \phi$. The deviation is positive if the indicated angle is too large. The separation of the pointer and the target is denoted by the angle $\zeta=\psi-\theta$, which is positive when the pointing direction is clockwise and negative when it is counterclockwise. The bisecting angle $\omega$ is used for the orientation of the stimuli.

angle $\phi$ relative to the line through the pointer and the origin. The angle $\phi$ is always positive and smaller than or equal to $180^{\circ}$. Instead of the actual pointer angle $\phi$, we will be mainly interested in the deviation from the veridical value. We will denote this deviation from the veridical by $\Delta \phi=\phi_{\text {measured }}-\phi_{\text {veridical }}$, which was positive if the subject pointed behind the target and vice versa. Also, we define the separation angle $\zeta=\psi-\theta$ as the angle between the pointer and the target and the orientation $\omega=1 / 2(\psi+\theta)$ as the angle of the bisector of the angle between the pointer and the target. It should be noted that $\zeta$ changes sign when pointer and target positions are interchanged. When. as seen from the observer. the pointer is to the left of the target. $\zeta$ is positive, and the pointing direction is clockwise with respect to the observer. If pointer and target positions are interchanged, $\zeta$ becomes negative, and the pointing is counterclockwise.

The subjects were positioned so that the turning point of the head was located directly above the origin. Also, the subjects were oriented in such a way that the direction of their gaze when looking straight ahead was aligned with $0^{\circ}$.

Each pointer and target was used at only one distance from the observer so that the apparent size was equal for all distances. The distances used $(1.47,2.10,3.00$, and $4.31 \mathrm{~m})$ were scaled with a factor of approximately $\sqrt{2}$. The pointers and targets were scaled accordingly (see Table 1).

\section{Procedure}

For each trial, the subjects were asked to direct a pointer so that it pointed straight to a given target. The position of the pointer and/or the target varied from trial to trial, whereas the vantage point was prescribed and the same for all measurements - that is, the origin of the polar grid (Figure 2A). Once the subject was satisfied with the setting, the pointing direction was noted. The subjects were given no feedback about their performance. The initial direction of the pointer was always the final direction of the previous trial. During the measurements, the subjects were allowed to turn their heads and move their eyes freely. However, lateral head movements were inhibited by the construction in the cabin.

The subject always entered the room blindfolded, and once seated in the cabin, the blindfold was removed. The same applied when the subject left the room. Hence, he or she was aware of the room from a single viewpoint only. During each trial, the experimenter was not visible; in between trials, no special precautions were taken, except that the subjects could never see the pointer and the target when they were being mounted.

Table 1

Pointer and Target Dimensions for Each Distance

\begin{tabular}{ccccc}
\hline Distance $(\mathrm{m})$ & $\begin{array}{c}\text { Target } \\
\text { Diameter }(\mathrm{cm})\end{array}$ & $\begin{array}{c}\text { Pointer } \\
\text { Length }(\mathrm{cm})\end{array}$ & $\begin{array}{c}\text { Thickness }(\mathrm{cm}) \\
\text { Disk }\end{array}$ & $\begin{array}{c}\text { Diameter }(\mathrm{cm}) \\
\text { Diane }\end{array}$ \\
\hline 1.47 & 2.2 & 12.2 & 0.5 & 4.0 \\
2.10 & 3.2 & 17.5 & 0.7 & 5.7 \\
3.00 & 4.5 & 25.0 & 1.0 & 8.2 \\
4.31 & 6.5 & 35.9 & 1.4 & 11.8 \\
\hline
\end{tabular}

Note-The pointer consisted of a rod and a disk. The diameter of the rod was the same as the thickness of the pointer. 
Table 2

Configurations Used in Experiment 1

\begin{tabular}{|c|c|c|c|c|c|c|c|c|c|}
\hline \multirow[b]{2}{*}{$|\zeta|$} & \multicolumn{3}{|c|}{$1.47 \mathrm{~m}, 2.10 \mathrm{~m}$} & \multicolumn{3}{|c|}{$3.00 \mathrm{~m}$} & \multicolumn{3}{|c|}{$4.31 \mathrm{~m}$} \\
\hline & $\mathrm{L}$ & M & $\mathrm{R}$ & $\mathrm{L}$ & M & $\mathbf{R}$ & $\mathrm{L}$ & M & $\mathbf{R}$ \\
\hline 15 & \pm & \pm & & \pm & \pm & & \pm & \pm & \\
\hline 30 & & + & - & & + & - & & + & - \\
\hline $45^{*}$ & \pm & \pm & \pm & \pm & \pm & \pm & & \pm & \\
\hline 60 & - & - & & - & - & & & \pm & \\
\hline 75 & & \pm & \pm & & \pm & & & & \\
\hline 90 & - & + & & & \pm & & & & \\
\hline 105 & & \pm & \pm & & & & & & \\
\hline 120 & + & + & & & & & & & \\
\hline 135 & \pm & \pm & & & & & & & \\
\hline 150 & & - & + & & & & & & \\
\hline 165 & \pm & \pm & & & & & & & \\
\hline 180 & & - & - & & & & & & \\
\hline 195 & & \pm & & & & & & & \\
\hline 210 & & \pm & & & & & & & \\
\hline
\end{tabular}

Note - In each entry, the sign of the values of the separation angle $\zeta$ is shown for each distance and orientation. A positive sign corresponds to pointing clockwise, and a negative sign to counterclockwise. If both configurations were measured, this is indicated with $\mathrm{a} \pm$. The letters $\mathrm{L}$, $M$, and $R$ denote configurations that are oriented to the left ( $\omega \geq 15^{\circ}$ ), middle $\left(-7.5^{\circ} \leq \omega \leq 7.5^{\circ}\right)$, and right $\left(\omega \leq-15^{\circ}\right)$, respectively. *Other orientations also were measured (see the text for details).

All the trials of both experiments were repeated three times. The trials in the monocular condition were presented first and consisted of three blocks: Each block was a single repetition of all the monocular trials, in randomized order. After that, the trials in the binocular condition were presented ordered in a similar way. During monocular measurements, all the subjects wore eyepatches over the left eyes. The trials were in random order, except that all the trials with a monocular viewing condition were measured first. The measurements were presented in sessions of approximately $1 \mathrm{~h}$. The total measuring time, including all 3 subjects, amounted to $60 \mathrm{~h}$ for 2,394 trials.

\section{EXPERIMENT 1}

In this experiment, the dependence of the pointer settings on the separation angle $\zeta$ was measured for several distances. We measured both monocularly and binocularly, in order to see what effect stereopsis would have on an exocentric pointing task. In fact, it was unclear whether the task could be done at all monocularly, since both the pointer and the target were scaled with distance so as to keep the apparent size constant. Considering that free turning of the head was allowed, one might argue that visual space should be isotropic: If a pointer-andtarget configuration is positioned in different orientations with respect to the observer, the subject only needs to turn his or her head in order to make the configurations identical. If this is the case, the pointer and the target positions will also be interchangeable, provided that the left and the right visual fields are symmetrical. Although we used this symmetry to reduce the number of measurements, these assumptions could still be verified. There were 200 different configurations, which were measured three times with subjects A.O., G.S., and J.N., resulting in a total of 1,800 trials.

\section{Method}

Monocular viewing condition. Both the pointer and the target were placed at the same distance from the observer. The distances used were 1.47 and $4.31 \mathrm{~m}$, and the stimuli were scaled accordingly. The absolute value of the separation angle $\zeta$ was varied from $15^{\circ}$ to $210^{\circ}$ in steps of $15^{\circ}$. However, at a distance of $4.31 \mathrm{~m}$, the maximum angle was restricted to $60^{\circ}$ because of the limited dimensions of the room (see Figure $2 \mathrm{~A}$ ). If the pointer and the target are interchangeable without affecting the results, only one of both configurations needs to be measured. Because we did not know whether this assumption was valid, we left out one configuration for half of the separation angles $|\zeta|$ (see Table 2). Consequently, we could still verify whether the pointer and the target positions are interchangeable (since interchanging the position corresponds to a change of sign of $\zeta$.

For each separation angle $\zeta$, one degree of freedom remains: The triangle spanned by the pointer, the subject, and the target can have different orientations $\omega$ (see Figure 2B). The number of positions was limited by measuring at only two orientations: straight ahead or to the side, either left or right (see Table 2). Only for a separation of $|\zeta|=45^{\circ}$ were all orientations measured: For a distance of 1.47 and $2.10 \mathrm{~m}$, these orientations were $-67.5^{\circ},-37.5^{\circ},-7.5^{\circ}, 22.5^{\circ}$, $52.5^{\circ}$, and $82.5^{\circ}$; for a distance of $3.00 \mathrm{~m},-22.5^{\circ},-7.5^{\circ}, 7.5^{\circ}$, and $22.5^{\circ}$; for a distance of $4.31 \mathrm{~m},-7.5^{\circ}$ and $7.5^{\circ}$. In this way, we were able to investigate whether visual space is indeed isotropic.

Binocular viewing condition. The configurations were the same as those in the monocular viewing condition, with the addition of the positions at a constant distance of 2.10 and $3.00 \mathrm{~m}$. For a distance of $2.10 \mathrm{~m}$, the same separation angles $\zeta$ and orientations $\omega$ were used as for $1.47 \mathrm{~m}$. For a distance of $3.00 \mathrm{~m}$, the same configurations were used as for $4.31 \mathrm{~m}$, except that the range of the separation $\zeta$ was extended to a maximum of $90^{\circ}$.

\section{Results}

In Figure 3, the results of the monocular and binocular viewing conditions are shown for all 3 subjects (A.O., G.S., and J.N.). All the data correspond to configurations in which both the pointer and the target were placed at a distance of $1.47 \mathrm{~m}$ from the subject. Each graph depicts the deviation $\Delta \phi$ of the indicated pointer direction from the veridical direction as a function of the separation angle $\zeta$. The deviation $\Delta \phi$ is the average of three repetitions, and the error bars denote the corresponding standard deviations. The different points for the same value of $\zeta$ correspond to the different orientations $\omega$ of the same configuration of the stimuli. The filled circles denote configurations that were placed directly in front of the subject $\left(\omega=0^{\circ}\right.$ or $\left.\omega= \pm 7.5^{\circ}\right)$, whereas the open diamonds and open boxes denote orientations that were oriented to the right $\left(\omega \leq-15^{\circ}\right)$ and to the left $\left(\omega \geq 15^{\circ}\right)$, respectively.

As is shown in the left column of Figure 3, there was an increasingly negative deviation $\Delta \phi$ as the separation angle $|\zeta|$ became small in the monocular viewing condition. When there was a negative deviation $\Delta \phi$, the subject actually pointed to a point somewhere between the target and himself, or in other words, there was an undershoot. This effect became large for small $|\zeta|$, meaning that the 
undershoot was large when the veridical direction was almost perpendicular to the line of sight (when looking at the pointer). This undershoot occurred despite the fact that the distance between the pointer and the target became very small $(38 \mathrm{~cm}$ at a distance of $1.47 \mathrm{~m}$ ).

The right column of Figure 3 shows the results for the binocular viewing condition. It is obvious that there were no large negative deviations $\Delta \phi$ in the binocular condition, which is in contrast to the monocular condition. Instead, there were smaller positive deviations. So, in general, one can say that the subjects performed better in an exocentric pointing task when they had binocular information. In Table 3 , the average deviation $(\overline{\Delta \phi})$ and the standard error of the mean $(S E)$ are shown for each subject and each distance. It can readily be seen that large negative deviations occurred only in the monocular condition. Only for subject G.S. and for a distance of $4.31 \mathrm{~m}$ was the average deviation positive. The average deviation $\overline{\Delta \phi}$ in the binocular condition was always positive for subjects A.O. and G.S. and approached zero for subject J.N. For subject A.O., the average deviations were relatively large (approximately $7^{\circ} \pm 1^{\circ}$ ) and larger than for the other 2 subjects.

When the left and right halves of the graphs in Figure 3 are compared, it is clear that, in general, the deviation is not independent of the sign of $\zeta$. Symmetry in the sign of $\zeta$ would mean that interchanging the positions of the pointer and the target would result in the same deviation of the indicated angle $\phi$, but this does not seem to have been the case. In order to look at the symmetries in more detail, it is useful to plot the orientation dependence for a fixed separation angle and distance of the pointer and the target.

In Figure 4, the deviation $\Delta \phi$ is shown for each subject as a function of the orientation $\omega$ for a pointer and target distance of $1.47 \mathrm{~m}$ with a separation of $|\zeta|=45^{\circ}$. The results for the other distances are summarized in Table 4. In each graph, two sets of points are shown: The diamonds correspond to pointing clockwise (with respect to the observer), and the stars correspond to pointing counterclockwise. The sign of the counterclockwise orientation $\left(\omega_{\mathrm{ccw}}\right)$ is flipped so that points belonging to configurations that are symmetrical in the $0^{\circ}$ line (see Figure 2) have the same $x$-axis value. As a result, an overlap of data corresponds to a visual field that is symmetrical with respect to the $0^{\circ}$ line, or in other words, visual space has a left/right symmetry with respect to the body. Note that this symmetry is not head-centric but body-centric, because the subject is allowed to turn his head freely. If the deviation $\Delta \phi$ were independent of the orientation (a zero slope), this would indicate that visual space is isotropic.

The left column of Figure 4 gives the results for each subject-A.O., G.S., and J.N.-in the monocular viewing condition. From the graphs it is clear that there is a considerable overlap between pointing clockwise (diamonds) and counterclockwise (stars). The average slope differs from zero, except for subject A.O. Furthermore, the slope is more or less the same for both pointing clock- wise and pointing counterclockwise, again with the exception of subject A.O.

The right column of Figure 4 shows the results for the binocular viewing condition. With regard to the binocular condition, three main aspects catch the eye: First, the slope is the same for pointing clockwise and counterclockwise; second, the slope is nonzero; and third, there is a considerable overlap of the data. The overlap is not perfect, however: it seems that for subjects A.O. and J.N., there is a positive and a negative bias, respectively, between pointing clockwise (diamonds) and pointing counterclockwise (stars). When the monocular condition is compared with the binocular condition, the average slopes are found to have a larger negative value in the binocular condition. Subject J.N. is an exception, since the slopes are about the same.

In order to test the significance of a slope difference and an offset difference between pointing clockwise and counterclockwise, we performed a multiple regression with interaction. For the pointing condition, an indicator variable was used so that the data could be fitted to the following equation: $\Delta \phi=a+b \cdot \omega+c \cdot \delta+d \cdot \omega \cdot \delta$, where $\delta$ is the dummy variable representing the pointing condition ( $\delta=1$ denotes clockwise, and $\delta=-1$ counterclockwise). The offset difference in $\Delta \phi$ is accounted for by the parameter $c$, whereas a slope difference comes from the interaction term with parameter $d$. From the multiple regression, two equations are obtained: $\Delta \phi=(a$ $+c)+(b+d) \cdot \omega$ for pointing clockwise and $\Delta \phi=(a-c)$ $+(b-d) \cdot \omega$ for pointing counterclockwise. From this it is readily seen that the offset difference equals $2 c$, the slope difference is $2 d$, and the average slope is $b$.

In Table 4 , the average slope, denoted by $\bar{b}$, the slope difference, denoted by $\Delta b$, and the offset difference, denoted by $\Delta a$, are shown with their significance levels. It is clear that there is no significant slope difference $\Delta b$, with only one exception (A.O., $2.10 \mathrm{~m}$, binocular condition). On the other hand, the offset difference $\Delta a$ is significant in many cases-that is, for subjects A.O. and J.N. in the binocular condition and for subjects G.S. and J.N. in the monocular condition. Thus, the asymmetry in the sign of $\zeta$, as observed in Figure 3 (which corresponds to an asymmetry between pointing clockwise and pointing counterclockwise), appears as an offset difference $\Delta a$ in Table 4.

In the monocular condition, the average slope is negative for all the subjects, although the average slope is approximately zero for subject A.O. (see Table 4). The value of $\bar{b}=0.90$ for subject G.S. at a distance of $4.31 \mathrm{~m}$ is an exception, but this may be due to the limited number of points: Only two orientations were measured (six data points for each pointing condition). A negative value for the average slope means that, for clockwise pointing, the deviation $\Delta \phi$ decreases to a large undershoot as the orientation of a configuration changes from right $(\omega=$ $\left.-90^{\circ}\right)$ to left $\left(\omega=90^{\circ}\right)$. Similarly, for pointing counterclockwise $\Delta \phi$ also decreases to a large undershoot, but now the orientation changes from left to right. Thus, in 

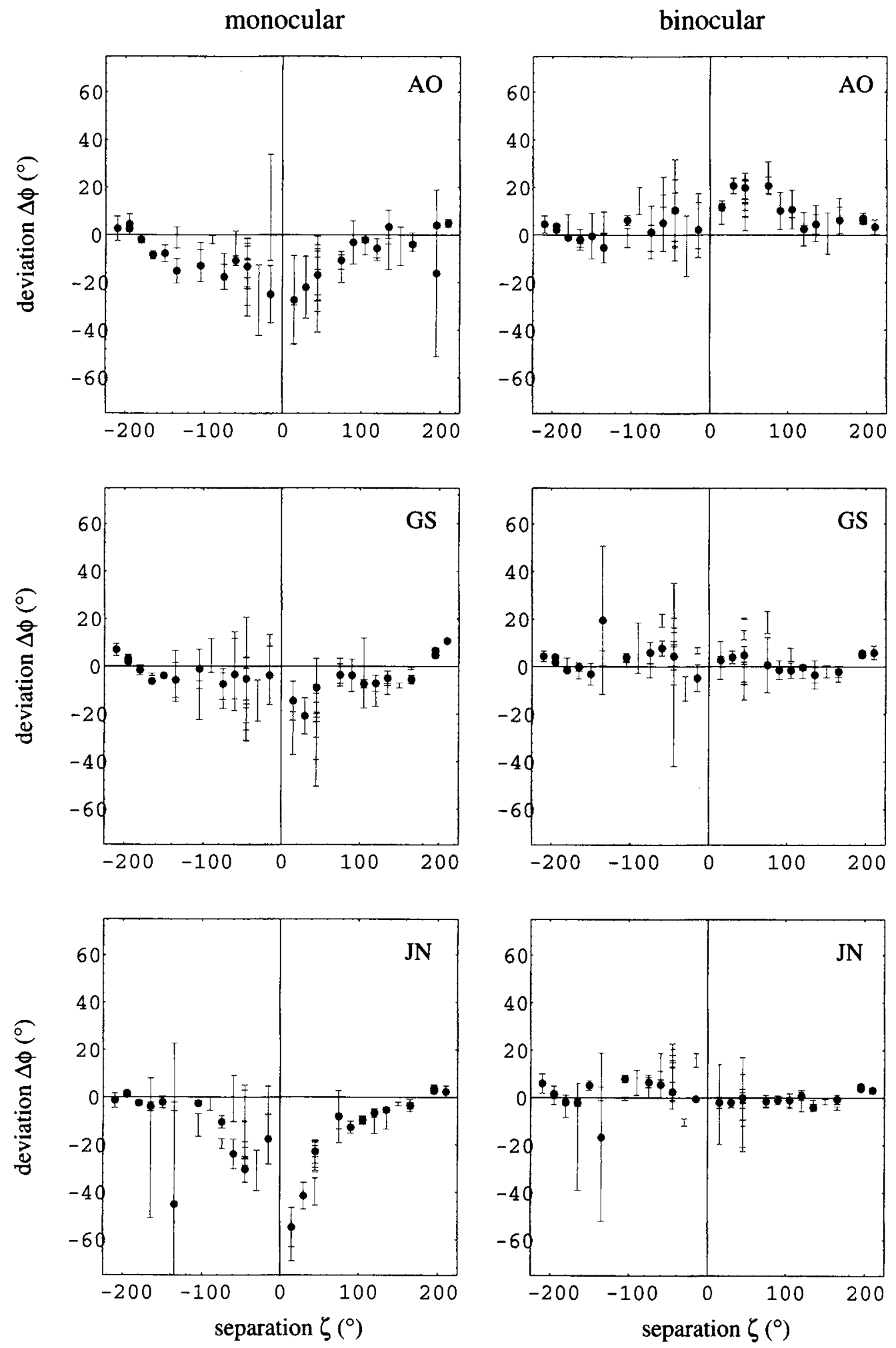

Figure 3. In each graph, the average deviation of the indicated angle $\phi$ from the veridical setting is shown as a function of the separation angle $\zeta$. The error bars indicate the standard deviations of three repetitions. The graphs in the left column show the monocular settings for subjects A.O., G.S., and J.N., whereas the graphs in the right column show the binocular settings. All the data correspond to configurations in which both the pointer and the target were placed at a distance of $1.47 \mathrm{~m}$ from the subject. The filled circles denote configurations that were placed in front of the subject, whereas the open diamonds and the open boxes denote configurations that were oriented to the right and to the left, respectively. 
Table 3

Average Deviation $\overline{\Delta \phi}$ of the Pointer Settings in Degrees for Each Subject

\begin{tabular}{ccccccc} 
& & \multicolumn{2}{c}{ Monocular } & & \multicolumn{2}{c}{ Binocular } \\
\cline { 7 - 8 } Subject & Distance $(\mathrm{m})$ & $\overline{\overline{\Delta \phi}}$ & $S E$ & & $\overline{\Delta \phi}$ & $S E$ \\
\hline A.O. & 1.47 & -10.3 & 1.0 & & 7.4 & 0.7 \\
& 2.10 & & & 4.3 & 1.0 \\
& 3.00 & & & 9.0 & 1.2 \\
G.S. & 4.31 & -0.2 & 2.6 & 8.0 & 2.2 \\
& 1.47 & -7.4 & 0.9 & 3.0 & 0.8 \\
& 2.10 & & & 1.8 & 0.8 \\
JN. & 3.00 & & & 3.6 & 0.7 \\
& 4.31 & 6.5 & 1.5 & 4.0 & 0.9 \\
& 1.47 & -15.1 & 1.4 & 0.8 & 0.8 \\
& 2.10 & & & -0.4 & 0.6 \\
& 3.00 & & & -1.5 & 0.8 \\
& 4.31 & -11.4 & 1.5 & -3.1 & 1.0 \\
\hline
\end{tabular}

Note-The results are shown for all distances in the monocular and binocular conditions. For distances of 1.47 and $2.10 \mathrm{~m}, 159$ measurements are averaged. For distances of 3.00 and $4.31 \mathrm{~m} .87$ and 45 measurements are averaged, respectively.

the monocular condition, visual space is anisotropic for subjects G.S. and J.N., since there is an orientation dependence, and in the case of subject A.O., the orientation dependence is in the same direction, although this is not significant.

The offset difference denoted by $\Delta a$ is negative throughout, although this is not significant in the cases of subject A.O. and, for a distance of $4.31 \mathrm{~m}$, subject J.N. An offset difference indicates that the left/right symmetry has a bias. But although the offset difference is significantly different from zero in the case of subjects G.S. and J.N., there is still a considerable overlap (see Figure 4, middle and bottom right graphs). So visual space is still approximately left/right symmetrical. Furthermore, for a distance of $4.31 \mathrm{~m}$, there were only two possible orientations, so the actual error may be larger than estimated.

For the binocular condition, the average slope $\bar{b}$ is negative and ranges from about -0.1 to -0.3 for all the subjects and for all the distances except $4.31 \mathrm{~m}$. At a distance of $4.31 \mathrm{~m}$, the values of $\bar{b}$ are even more negative. On the other hand, the regression lines were calculated for only two orientations $\omega$, so the error may be much larger than estimated. In most cases, the average slope is more negative than was found for the monocular viewing condition. This would indicate that the anisotropy is stronger in the binocular case.

The difference between the offsets for pointing clockwise and for pointing counterclockwise $(\Delta a)$ in the binocular viewing condition turns out to be small but significant for subjects A.O. and J.N. ( $p=.1$ for subject A.O. at a distance of $4.31 \mathrm{~m}$ and $p<.004$ otherwise). We find values of approximately $10^{\circ}, 0^{\circ}$, and $-10^{\circ}$ for A.O., G.S., and J.N., respectively. Thus, only subject G.S. appears to have a good left/right symmetry, whereas subjects A.O. and J.N. show small biases that are consistent over all distances but opposite in sign.

\section{Discussion}

Although the results obtained in the monocular viewing condition are different from those in the binocular viewing condition, the settings of each subject do not, as one might expect, become independent of the stimulus positions. Thus, the environment must have contained sufficient monocular cues for the subjects to carry out the task. This is somewhat surprising, because the apparent sizes of the pointer and the target were kept constant. Also, monocular cues from the floor, walls, and ceiling were reduced as much as possible (without dimming the lights). Subjects A.O. and J.N. reported having noted only two and three different sizes, respectively, of the pointer and the target, whereas actually four were used. Subject G.S. had no trouble at all in distinguishing the stimuli. A possible explanation for this is that the pointer could rotate and that both the pointer and the target could sway slightly. Furthermore, the subjects could rotate their heads freely, although lateral movements were inhibited. All these movements contribute to a motion parallax that a subject can use in coming to a decision during an experiment. Another explanation is that the subjects may have been able to derive information from observing the experimenter's movements. There is no indication that this was the case, because the subjects behaved very similarly and did not notice the number of different pointers. At best, the subjects were able to aim the pointer in the right ballpark.

When the pointer and the target were placed at the same distance, stereopsis improved the veridicality of the judgments of the subjects. The large undershoot observed in the monocular case was no longer present in the binocular case; subject A.O. even showed an overshoot. The degree of mispointing depended both monocularly and binocularly on the separation between the pointer and the target: the smaller the separation, the larger the deviation of the pointer direction from the physically correct direction.

A rather straightforward explanation would be that when the separation angle $|\zeta|$ was large, an error in estimating the pointer and target distance had only a small effect on the pointing direction. When the subject was situated between the pointer and target $\left(|\zeta|=180^{\circ}\right)$, the pointing direction $\left(\phi=0^{\circ}\right)$ would have been the same irrespective of the distance between the subject and the pointer and the subject and the target. On the other hand, this is somewhat surprising because, for angles $|\zeta|>90^{\circ}$, the subject could no longer see the pointer and the target at the same time. Only by turning the head back and forth can he or she make a judgment. Apparently, the accuracy did not suffer from this additional complication, and the subjects seem to have been able to judge egocentric directions well.

The fact that the deviation $\Delta \phi$ depended linearly on the orientation of a given pointer-and-target configuration reveals that binocular visual space was anisotropic for all the subjects. This effect was weaker in the monocular 
monocular
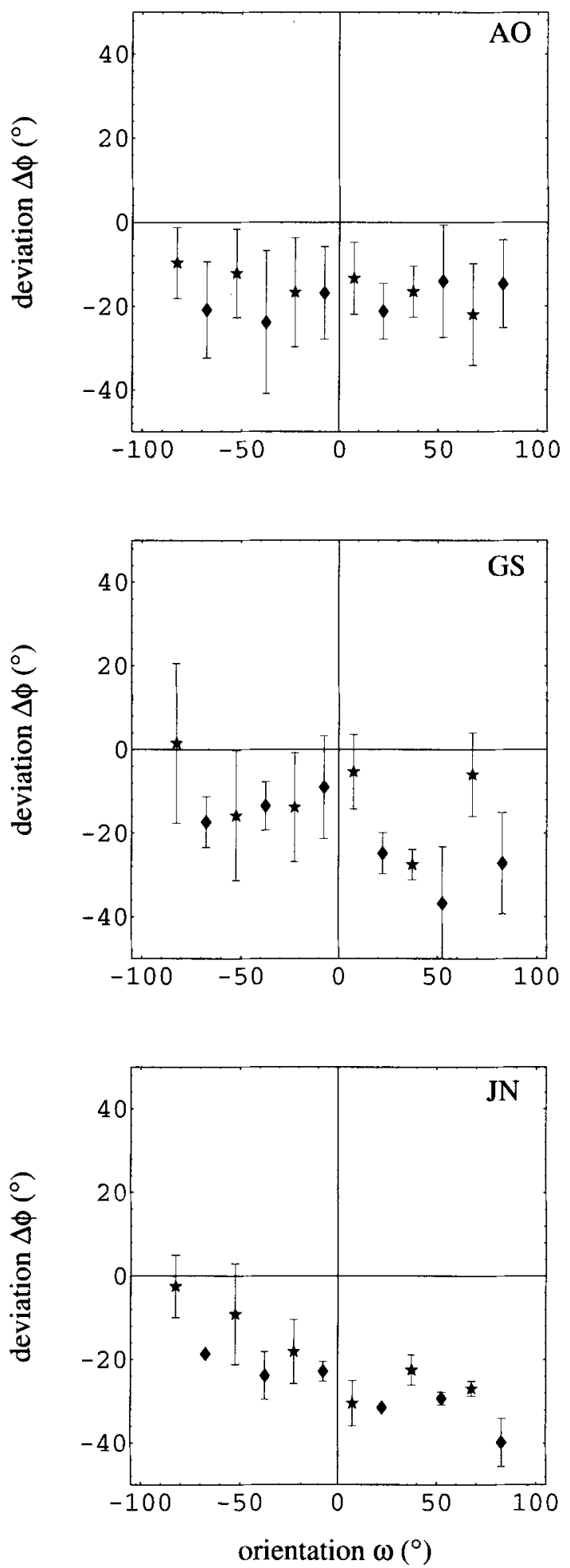

binocular
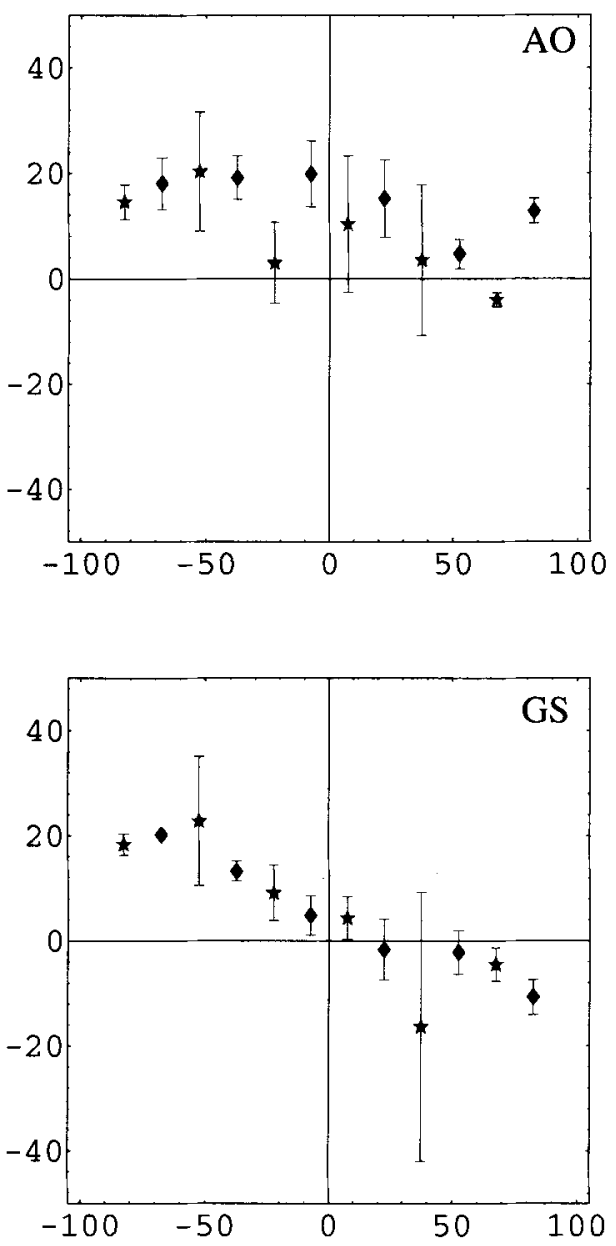

Figure 4. In each graph, the average deviation of the indicated angle $\phi$ from the veridical setting is shown as a function of the orientation angle $\omega$. The error bars denote the standard deviation of three repetitions. The graphs in the left column show the monocular settings for subjects A.O., G.S., and J.N., whereas the graphs in the right column show the binocular settings. All the data correspond to configurations in which both the pointer and the target were placed at a distance of $1.47 \mathrm{~m}$ from the subject. The diamonds correspond to pointing clockwise, and the stars to pointing counterclockwise.

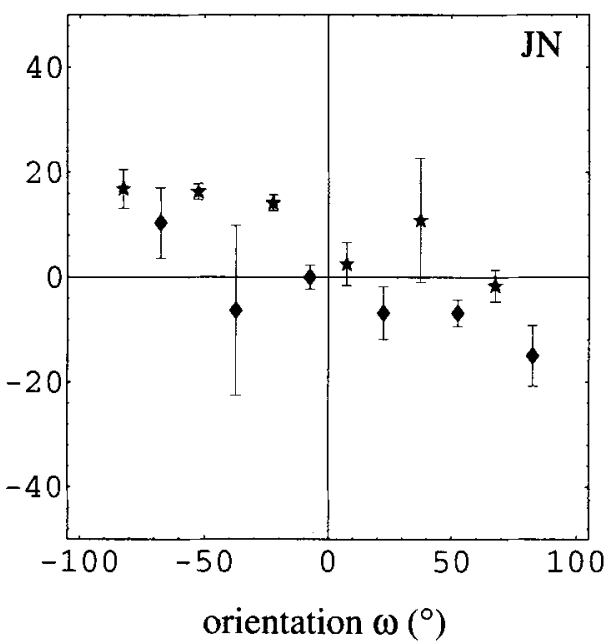


Table 4

For Each Subject and Distance, the Average Slope $(\vec{b})$, the Slope Difference $\Delta b$, and the Difference in the Offset $(\Delta a)$ for Pointing Clockwise and Counterclockwise

\begin{tabular}{|c|c|c|c|c|}
\hline Subject & Distance (m) & $\Delta a$ & $\Delta b$ & $\bar{b}$ \\
\hline \multicolumn{5}{|c|}{ Monocular } \\
\hline \multirow[t]{2}{*}{ A.O. } & 1.47 & -3.4 & 1.9 & -0.01 \\
\hline & 4.31 & -13.2 & -0.4 & -0.03 \\
\hline \multirow[t]{2}{*}{ G.S. } & 1.47 & $-8.9^{*}$ & -0.8 & $-0.09^{*}$ \\
\hline & 4.31 & $-8.0^{*}$ & 0.1 & $0.90 \dagger$ \\
\hline \multirow[t]{2}{*}{ J.N. } & 1.47 & $-7.2 \dagger$ & 1.0 & $-0.15 t$ \\
\hline & 4.31 & -6.4 & 1.0 & -0.16 \\
\hline \multicolumn{5}{|c|}{ Binocular } \\
\hline \multirow[t]{4}{*}{ A. 0 . } & 1.47 & $8.5+$ & 1.1 & $-0.10 \dagger$ \\
\hline & 2.10 & $12.9 \dagger$ & $2.2^{*}$ & -0.26 \\
\hline & 3.00 & 10.97 & -0.7 & -0.07 \\
\hline & 4.31 & 14.3 & 0.8 & -0.70 \\
\hline \multirow[t]{4}{*}{ G.S. } & 1.47 & 1.5 & 0.5 & $-0.21+$ \\
\hline & 2.10 & $-7.2^{*}$ & -1.9 & $-0.16 \dagger$ \\
\hline & 3.00 & 0.8 & 1.1 & $-0.42 \dagger$ \\
\hline & 4.31 & -1.8 & -0.3 & $-0.87 \dagger$ \\
\hline \multirow[t]{4}{*}{ J.N. } & 1.47 & $-12.1+$ & -0.3 & $-0.12 \dagger$ \\
\hline & 2.10 & $-12.0 \dagger$ & 0.1 & $-0.08 \uparrow$ \\
\hline & 3.00 & $-9.0 \dagger$ & -0.7 & $-0.19 t$ \\
\hline & 4.31 & $-10.9 t$ & 0.0 & $-0.31 *$ \\
\hline
\end{tabular}

Note--The slopes and offsets are calculated using multiple regression with interaction (see the text for details). The slope values and slope difference values are dimensionless, whereas the offset values are in degrees. For each condition, the parameter values are calculated from 18 measurements for a distance of 1.47 and $2.10 \mathrm{~m}$ and from 12 and 6 measurements for a distance of 3.00 and $4.31 \mathrm{~m}$, respectively. The significance levels are indicated with $a^{*}$ for $p<.05$ and $a^{+}$for $p<.01$.

condition, where there was no clear orientation dependence for subject A.O. Apparently, the viewing condition affects visual space quite profoundly, since its symmetries can differ.

The cause of such an anisotropy is unclear. Perhaps the orientation dependence is related to the stance of the eyes in the orbit. But any asymmetry caused by this is with respect to the head. This, in itself, cannot explain why there is an orientation dependence. Additional assumptions are needed concerning the strategy a subject adopts in executing the pointing task. It was observed that the subjects used a lazy approach while directing the gaze back and forth between the pointer and the target: They only started to turn their heads when moving their eyes further was no longer possible without reducing the binocular image quality. The turnover is near $30^{\circ}$ to the left or right, so the binocular visual field subtends an angle of $60^{\circ}$ with respect to the head. Beyond this point, a subject starts looking against his or her own nose, which results in a monocular image, and consequently, the subject will turn his or her head. If the subject uses a lazy-head strategy, the pointer and the target will be perceived differently if they are oriented to the right (or left). As a consequence, a difference occurs between pointing clockwise and pointing counterclockwise, because the pointer is oriented more to the right of the observer when pointing counterclockwise than when pointing clockwise.

Deviations in the binocular condition of left/right symmetric configurations of the pointer and the target were approximately the same. This indicates that visual space is approximately left/right symmetrical. Two subjects had a bias of about $10^{\circ}$, which was positive in one case and negative in the other. This bias was independent of the distance and, therefore, probably reflected a property of the subject. In the monocular case, there did not appear to be such a constancy.

An explanation for the left/right asymmetry that was found for 2 subjects might be eye dominance. When a subject directs his gaze back and forth from pointer to target, it matters whether the pointer (and the target) is positioned to the left or to the right with respect to the head of the subject. This difference could have lead to the measured bias. However, the actual mechanism underlying this effect is not at all clear. Alternatively, one might argue that the symmetry axis of the subject was misaligned with the $0^{\circ}$ line. This would appear as a horizontal shift in Figure 4, rather than as a vertical shift. However, an offset difference of $10^{\circ}$ in $\Delta \phi$ would require a misalignment of $\Delta \omega \approx 50^{\circ}$, because the average slope of the measured deviation $\Delta \phi$ as a function of the orientation $\omega$ was about 0.2 . Such a large misalignment does not seem likely.

\section{EXPERIMENT 2}

The logical counterpart of Experiment 1 was to vary the relative distance of the pointer and the target from the observer. This may provide insight into the distance dependence of the pointing task, and consequently, questions about the depth structure of visual space can be addressed. Also, we wanted to verify that the stereopsis cue was actually used by the subject; we could do this by comparing the data with the monocular condition. Furthermore, it would be interesting to see whether the task is in fact possible when viewing monocularly.

\section{Method}

In this experiment, the angular separation and the orientation were kept constant, whereas the relative distance of the pointer and the target with respect to the observer was varied. As before, the stimuli were scaled accordingly. The orientation was set to straight ahead (i.e., $\omega=0^{\circ}$ ), and the measurements were done for three separation angles $\zeta$ of $30^{\circ},-60^{\circ}$, and $90^{\circ}$. All possible distances were used-that is, from 1.47 to $4.31 \mathrm{~m}$ in the case of a separation of $30^{\circ}$ and $-60^{\circ}$ and from 1.47 to $3.00 \mathrm{~m}$ for a $90^{\circ}$ separation. The range was smaller for a separation of $90^{\circ}$ because of the limited dimensions of the room. All the trials were carried out both monocularly and binocularly and were repeated three times. The trials were presented in random order and mixed with those of Experiment 1 , although the trials in the monocular condition were presented first. There were 82 different trials, which were reduced to 66 trials owing to a small overlap with Experiment 1. For 3 subjects (A.O., G.S., and J.N.) and three repetitions, this yielded a total of 594 additional trials.

\section{Results}

In Figure 5, the results are shown for the monocular viewing condition. In each graph, the deviation $\Delta \phi$ of the pointer direction is plotted as a function of the relative distance $r_{\mathrm{p}} / r_{\mathrm{t}}$ of the pointer and the target (with respect to 

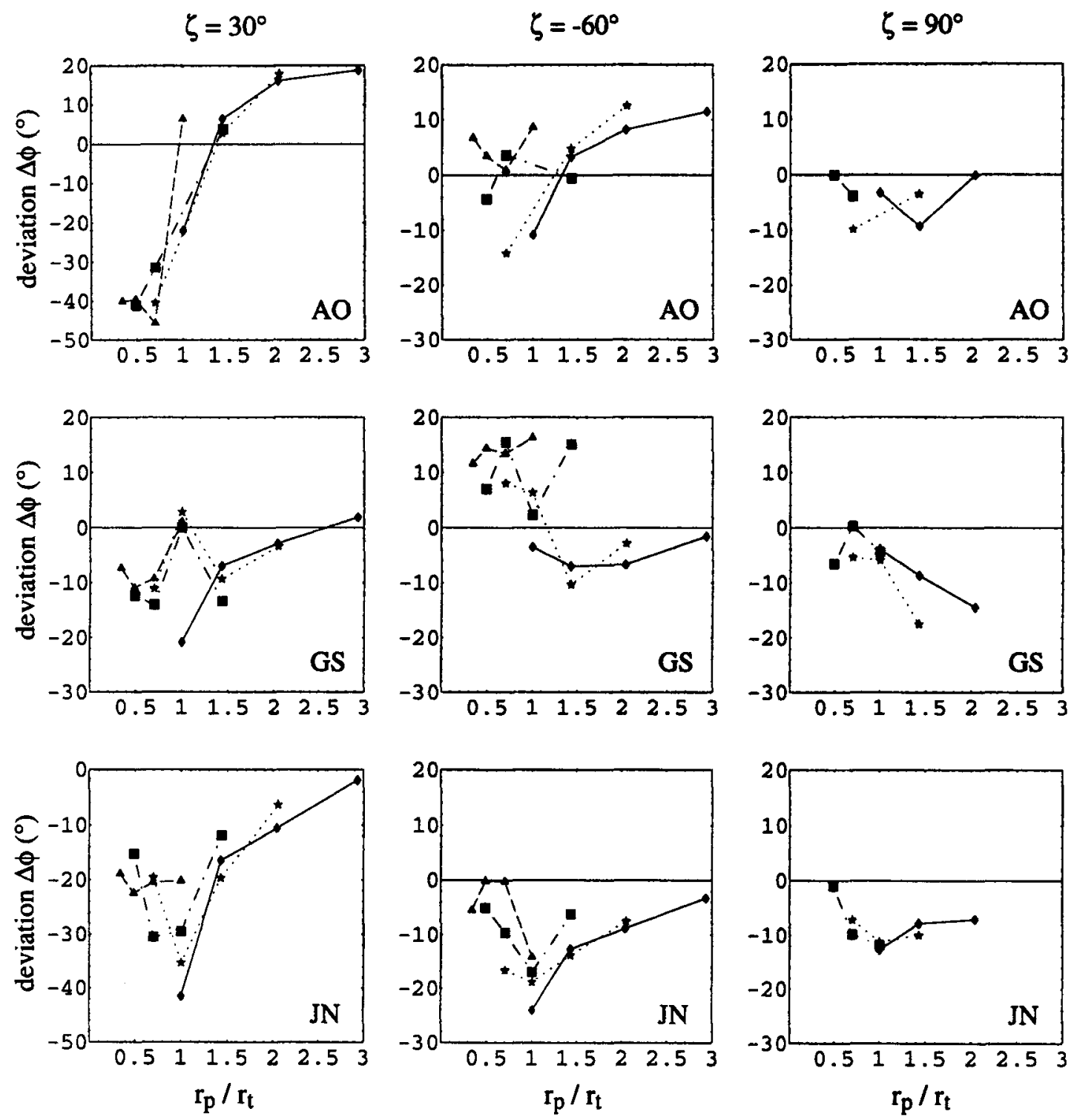

$$
\begin{array}{lll}
\mathrm{r}_{\mathrm{t}}=1.47 \mathrm{~m} & \cdots- & \mathrm{r}_{\mathrm{t}}=3.00 \mathrm{~m} \\
\mathrm{r}_{\mathrm{t}}=2.10 \mathrm{~m} & -\cdots & \mathrm{r}_{\mathrm{t}}=4.31 \mathrm{~m}
\end{array}
$$

Figure 5. Each graph depicts, for the monocular viewing condition, the deviation of the indicated angle $\phi$ from the veridical setting as a function of the ratio between the pointer distance $r_{p}$ and the target distance $r_{\mathrm{r}}$. The line styles and markers correspond to the different target distances: The solid line with diamonds corresponds to $r_{t}=1.47 \mathrm{~m}$, the dotted line with stars to $r_{\mathrm{t}}=\mathbf{2 . 1 0} \mathrm{m}$, the dash-dotted line with squares to $r_{\mathrm{t}}=\mathbf{3 . 0 0} \mathrm{m}$, and the dashed line with triangles to $r_{\mathrm{t}}$ $=4.31 \mathrm{~m}$. The rows show the results for Subjects A.O., G.S., and J.N., respectively. Each column corresponds to a different separation angle: From left to right, the separation angles are $30^{\circ},-60^{\circ}$, and $90^{\circ}$.

the observer). Each line connects the data points of four pointer distances $r_{\mathrm{p}}$ for a single target distance $r_{\mathrm{t}}$. The top, middle, and bottom rows represent the data for subjects A.O., G.S., and J.N., respectively. In the left, middle, and right columns, the separation angle $\zeta$ is $30^{\circ},-60^{\circ}$, and $90^{\circ}$, respectively.

From the graphs in Figure 5, it can be seen that there is a reasonable overlap between the various lines when it is taken into account that the standard error of the mean
(SEM) of each point is, on average, $5^{\circ}$. This is especially clear in the top left (subject A.O., $\zeta=30^{\circ}$ ) and bottom right (subject J.N., $\zeta=90^{\circ}$ ) graphs. It is difficult to find a common trend in the results for the various subjects. In fact, the subjects show large differences especially for $\zeta=30^{\circ}$. There are slight similarities between the separation angles, although the range of the deviations decreases as the separation angle increases. This is similar to the results of Experiment 1 , in which it was also observed that 
the deviations $\Delta \phi$ decreased as the separation $|\zeta|$ of the pointer and the target increased. For subject J.N., the shape of the curves appears very similar: They all have a minimum at $r_{\mathrm{p}} / r_{\mathrm{f}}=1$ and tend to zero for both smaller and larger values. Only the magnitude of the deviations $\Delta \phi$ is different. Most of the time, the pointer settings were far from veridical $\left(\Delta \phi=0^{\circ}\right)$ : Mispointings as large as $40^{\circ}$ undershoot occurred. Also, pointing in the frontoparallel plane $\left(r_{\mathrm{p}} / r_{\mathrm{t}}=1\right)$ did not generally result in veridical pointing; only subject G.S. showed such a tendency.

Figure 6 shows the results for the binocular viewing condition. Clearly, there is a considerable overlap in the data: The average $S E M$ amounts to approximately $2^{\circ}$, which is considerably smaller than that in the monocular condition. Furthermore, there is a clear dependence of the deviation $\Delta \phi$ on the relative distance $r_{\mathrm{p}} / r_{\mathrm{t}}$. In each graph (except for the top left graph), there is an overshoot $(\Delta \phi>0)$ when the pointer was closer to the observer than was the target $\left(r_{\mathrm{p}} / r_{\mathrm{t}}<1\right)$, whereas there is an undershoot $(\Delta \phi<0)$ when the pointer was further from the observer than was the target $\left(r_{p} / r_{t}>1\right)$. When the pointer and the target were placed at the same distance from the observer $\left(r_{\mathrm{p}} / r_{\mathrm{t}}=1\right)$, the settings were almost veridical, in contrast to the monocular condition. Only subject A.O. for a separation angle of $\zeta=30^{\circ}$ showed a large deviation of about $20^{\circ}$ (top left graph), when it should have been frontoparallel. A comparison of the 3 subjects reveals that the shape of the graphs is indeed very similar, but again the top left graph is an exception. When the different separation angles are compared, the dependence of the deviation $\Delta \phi$ on the relative distance $r_{\mathrm{p}} / r_{\mathrm{t}}$ is also very similar. This invariance of subject and separation angle is different from the monocular viewing condition.

\section{Discussion}

The results show clearly that the pointer settings did not depend on the absolute distance, in particular for the binocular condition. For the monocular case, this would not be so surprising, because the visual angle of the stimuli was kept constant. As a consequence, configurations of the stimuli with the same relative distance but at different absolute distances will be indistinguishable, provided no other cues (such as accommodation) interfere. In the binocular case, however, disparity and vergence provided information on the absolute depth, although the accuracy was rather poor, as compared with relative disparity (Collewijn \& Erkelens, 1990). Nonetheless, the data depended only on the relative distance, which indicates that knowledge about the absolute distance, if present, was not being used for this range of distances.

From the results, it is also clear that the pointer settings depended strongly on the relative distance $r_{p} / r_{t}$, in both the monocular and the binocular conditions. As was discussed in Experiment 1, this is surprising for the monocular condition, because the visual angle of the stimuli was kept constant and external reference lines of the floor, walls, and ceiling were removed as much as possible. Motion parallax and accommodation were still pos- sible cues for distance, which may explain why there was a dependence. Unfortunately, the experimenter's movements were visible between trials, from which information about the position of the stimuli could have been derived as well. Only for subject J.N. is there an indication that this information was actually used, because he systematically aimed at a spot before the target. However, subject J.N. did not aim the pointer straight at the position at which the experimenter was visible: The deviation increased with the distance of the target. Apart from this, subject J.N. was not very different from the other 2 subjects. So, even if the experimenter's movements were used by J.N., this does not affect the main conclusions.

In the monocular condition, the range of the deviations $\Delta \phi$ decreased as the separation angle $|\zeta|$ increased, whereas there was no such dependence in the binocular case. This is similar to the results of Experiment 1, where we found a vanishing deviation when the separation angle approached $|\zeta|=180^{\circ}$. Again, one might ascribe this effect to the insensitivity of the pointing task for inaccurate depth estimates when the separation angle is large. In Experiment 1 the same applied for the binocular condition, albeit to a lesser degree, but in Experiment 2 the pointer settings did not depend on the separation angle. Possibly, this effect is too small for the binocular viewing condition. The deviations became very large (up to $-40^{\circ}$ ) for a separation angle of $30^{\circ}$, which indicates that the task was very difficult in the monocular condition. Also, the differences between the subjects were considerable. Possibly, the subjects developed different strategies in order to cope with the difficulty of the task.

The differences between the subjects were large in the monocular condition, but they were very small in the binocular case. Apparently, stereopsis improved space perception, in that the standard error was reduced and the consistency across subjects and separation angles improved. Nonetheless, stereopsis did not generally improve the veridicality. Only when the pointer and the target were at the same distance from the observer were the settings veridical.

\section{GENERAL DISCUSSION AND CONCLUSIONS}

Although it is commonly assumed that, with stereopsis, depth perception is more veridical, this did not really manifest itself in the size of the deviations measured in the pointing task. In the monocular condition, the deviations were large, but the pointer settings were not independent of the stimulus positions, even though the visual angle of the stimuli remained constant and any reference from the walls, floor, and ceiling was minimized. In the binocular condition, on the other hand, the deviations of the pointer settings from the veridical were also large. Only when the pointer and the target were placed directly in front of the subjects and at the same distance from the observer were the settings nearly veridical, which is in contrast to the monocular viewing condition. Stereopsis, 

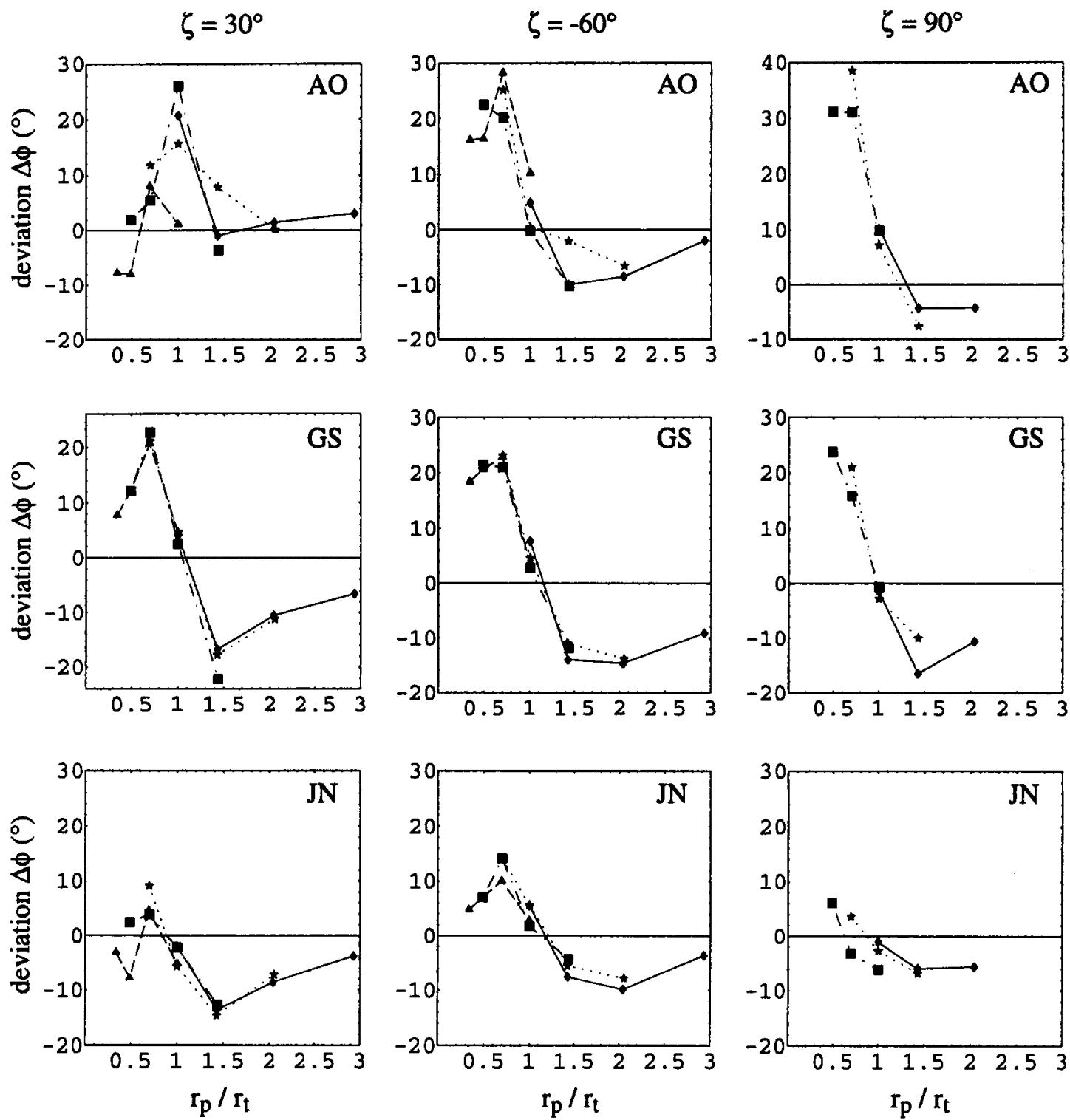

$$
\begin{array}{llll}
\mathrm{r}_{\mathrm{t}}=1.47 \mathrm{~m} & -\mathrm{r}_{\mathrm{t}}=3.00 \mathrm{~m} \\
\mathrm{r}_{\mathrm{t}}=2.10 \mathrm{~m} & - & \mathrm{r}_{\mathrm{t}}=4.31 \mathrm{~m}
\end{array}
$$

Figure 6. Each graph depicts, for the binocular viewing condition, the deviation of the indicated angle $\phi$ from the veridical setting as a function of the ratio between the pointer distance $r_{\mathrm{p}}$ and the target distance $r_{\mathrm{t}}$. The line styles and markers correspond to the different target distances: The solid line with diamonds corresponds to $r_{t}=1.47 \mathrm{~m}$, the dotted line with stars to $r_{\mathrm{t}}=\mathbf{2 . 1 0} \mathrm{m}$, the dash-dotted line with squares to $r_{\mathrm{t}}=\mathbf{3 . 0 0} \mathrm{m}$, and the dashed line with triangles to $r_{1}=4.31 \mathrm{~m}$. The rows show the results for subjects A.O., G.S., and J.N., respectively. Each column corresponds to a different separation angle: From left to right, the separation angles are $30^{\circ},-60^{\circ}$, and $90^{\circ}$.

however, did affect the consistency of the measurements, not only for each subject, but also across subjects. In the literature, there are only a few studies that compare monocular and binocular cues. For example, Foley (1977) compared verbal and manual distance estimates in monocular, binocular, and multicue conditions for a distance range of $11-33 \mathrm{~cm}$. Similar to the results reported here, it was found that the perceived distance depended systematically on the physical distance in the monocular condition and that there were considerable deviations in the binocular condition. Only in the multicue condition did the deviations from the veridical vanish.

The consistent picture for the binocular viewing condition in Experiment 2 suggests that a distance function may exist that describes the data. An important constraint on possible distance functions can already be obtained from the data of Figure 6: Because there was an overshoot when the pointer was nearer the subject than was the tar- 
get and vice versa, it can be shown that any distance function $d(r)$ must have an expanding part when the pointer is nearer and a compressing part when the target is nearer. More formally, if the deviation $\Delta \phi<0$, then the distance function must satisfy $d^{\prime \prime}(r)<0$, and the other way around, if $\Delta \phi>0$, then $d^{\prime \prime}(r)>0$. On these grounds, a large class of functions can already be rejected. For instance, for a power law the second derivative is either negative or positive, depending on the exponent. A number of distance functions have been proposed in the literature, such as the function proposed by Luneburg (1947), Gilinsky (1951), and Foley $(1980,1991)$. The second derivative of Gilinsky's function $d(r)=a \cdot r /(a+r)$ is $d^{\prime \prime}(r)<0$ for all $r>$ 0 . Thus, it has no expanding part and, therefore, cannot explain an overshoot of $\Delta \phi>0$. Foley (1991) proposed a model in which distance is perceived relative to a reference point but the reference distance is misjudged. If we assume that the target is the reference, the perceived pointer distance $d(r)$ equals Gilinsky's function, with $a=$ $I r_{\mathrm{t}} /\left(E r_{\mathrm{t}}+(F-1) I\right)$ (see Foley, 1991, for details). Therefore, this function does not apply, for the same reason as Gilinsky's function. The second derivative of the Luneburg function $d(r)=e^{-\mu}$ can be both positive and negative-that is, $d^{\prime \prime}(r)>0$ if $r<a / 2$, and $d^{\prime \prime}(r)<0$ if $r>$ $a / 2$. So, qualitatively, this function provides a better description. The same conclusion was already drawn by Koenderink and van Doorn (1998). But for a quantitative description, there is a serious drawback: The parameter $a$ in the Luneburg function is already determined by a single point. As a result, a numerical fit will be difficult, because there is no parameter left that controls the rate of compression and expansion.

It is interesting to see how the error in the pointer settings compares with the thresholds of monocular and binocular vision. In the monocular condition, the discrimination of the pointer direction is determined by the length of the projection of the pointer on the retina. Turning the pointer over a small angle changes the length of this projection. In the monocular condition, the average standard deviation of $\overline{S D}=7^{\circ}$ corresponded to an average change of 46 arcminutes in the visual angle.

In the binocular condition, there is also stereo information present - that is, changing the direction of the pointer causes a change in absolute disparity of the tip. The rate of change also depends on the distance. In this case, an average standard deviation of $\overline{S D}=5^{\circ}$ corresponded to a change of 47 arcseconds in absolute disparity for a distance of $1.47 \mathrm{~m}$. This is much smaller than the average change in the visual angle, which was 33 arcminutes for $\overline{S D}=5^{\circ}$. For the distances $2.1,3.0$, and $4.31 \mathrm{~m}$, the average disparities were 33,23 , and 16 arcseconds, respectively. These values are of the same order as the discrimination thresholds for stereoacuity.

The relative disparity between the tip and the end of the pointer also changes as a function of the pointer direction. For a distance of $1.47 \mathrm{~m}$ and an average standard deviation of $\overline{S D}=5^{\circ}$, the average change in relative disparity amounted to 1.46 arcminutes, which is about three times as large as the change in absolute disparity. For the distances $2.1,3.0$, and $4.31 \mathrm{~m}$, the average disparities were $1.02,0.71$, and 0.50 arcminutes, respectively.

Thus, the change in the visual angle of the pointer was, at least geometrically, the most important cue for discrimination of the pointer direction in both the monocular and the binocular conditions. The measured average standard deviations are well above acuity thresholds and are therefore easily discriminable. Furthermore, because the visual angle of the stimuli was kept constant, the (monocular) discriminability was independent of the distance. In the binocular condition, disparity provided additional cues for the direction of the pointer. These cues were much harder to detect, however, and the differences in absolute disparity were already near discrimination thresholds of stereo acuity.

It is conceivable that, instead of the relative distance, the direction of the pointer is misperceived. So the measured deviations from veridical can be described as a deformation of either the perceived distance or the perceived direction of the pointer. Formally, these descriptions are equivalent for a given separation angle. However, each description predicts a different dependence of the measured deviations $\Delta \phi$ on the separation angle $\zeta$ : If we take the relative distance equal to $r_{\mathrm{p}} / r_{\mathrm{t}}=1$, we would expect that the deviations would be zero for each separation angle if the perception of the distance was distorted, whereas one would expect a dependence if the perception of the pointer direction was distorted. In Experiment 1, it was found that, in the binocular condition, the deviation $\Delta \phi \approx$ 0 was independent of the separation angle, suggesting that only the distance was distorted. But in the monocular condition, there was a clear dependence, suggesting that the perception of the pointer direction was distorted. However, as was argued before, the strongest cues for perception of the pointer direction are monocular, so why would the direction be perceived differently in the monocular and in the binocular conditions? By assuming that the distance is distorted, the results of Experiment 1 in the binocular condition are obtained automatically. The results for the monocular condition are obtained if the distance of the pointer is overestimated relative to the target distance. This is plausible, because in the monocular condition distance estimates are very poor.

Thus far, the perception of the pointer direction and the distance are treated as independent. It is very likely, however, that they are not independent. For example, if the distance is perceived distortedly, the distance of each tip of the pointer is also perceived distortedly and, consequently, so is the direction of the pointer. From this point of view, there is no ambiguity between a distorted perception of the pointer direction and a distorted perception of the distance. Both arise from the same phenomenon: a distorted visual space. The question that is unanswered and that requires further analysis is whether there exists a consistent single deformed visual space that explains all the data. Although we think it is unlikely that the perception of the distance and the perception of the pointer 
direction are independent, more evidence is needed to prove or disprove the existence of a single consistent deformed visual space.

In between trials, the movements of the experimenter were visible. This is unfortunate, because this might be the reason why subjects were able to "guess" the correct direction of the pointer in the monocular condition, apart from motion parallax and accommodation. It seems that only subject J.N. may have used this information in the monocular condition. However, subject J.N. does not differ from the other subjects: The same conclusions apply for subject J.N. as for the other subjects.

In conclusion, we found that stereopsis provides additional information and is also used in an exocentric pointing task. Monocularly, the task is much harder, but the pointer settings still depend systematically on the stimulus positions. The main differences between monocular and binocular viewing are that the standard deviation becomes smaller and the results are more consistent in the binocular condition. Nevertheless, the deviations remain large in the binocular condition. Similar results were found earlier by Foley (1977).

It was found that the deviations depend linearly on the orientation of the stimulus configuration, indicating that visual space is anisotropic. At the same time, the deviations of left/right symmetrical configurations are approximately the same. This indicates that visual space is approximately left/right symmetrical. Subjects A.O. and J.N. did show a small but significant bias, however. Also, the pointer settings depend on the separation angle of the stimuli: The deviations from the veridical vanished for large angles. This may be explained by the insensitivity of the pointing task for misjudging distances for these large angles. Several studies report the failure of the left/right symmetry for a few individual subjects when the head is held fixed (Foley, 1972; Heller, 1997b). The unequal magnification of the lenses of the two eyes, or aniseikonia, is mentioned as a possible cause. Because in most of these studies the head of the subject was fixed, it cannot be compared directly with the anisotropy found in the present results. An exception is Battro et al. (1976), in which asymmetries were found even when the subject was allowed to turn his head freely.

The relative distance proves to be the most relevant parameter as far as the depth structure of visual space is concerned. The results for the different absolute distances of the pointer and the target coincide for the range of $1.47-$ $4.31 \mathrm{~m}$. The consistent picture in the binocular case across subjects and separation angles also suggests the existence of a distance function of some sort. A necessary constraint is that it must have an expanding part when the pointer is positioned closer to the subject than is the target and a compressing part if it is positioned further away. It turns out that of two common functions in the literature, Luneburg's (1947) function satisfies this requirement, whereas Gilinsky's function docs not. This is in agreement with the findings of Koenderink and van Doorn (1998).
Although there are many differences among subjects at a detailed level, the general picture is indeed very similar, especially in the binocular condition. All the subjects use stereopsis, if available, to determine the correct pointer direction. Nonetheless, the deviations from the veridical remain large, but they become very systematic. On the whole, the left/right symmetry of visual space is maintained, but at the same time it is strongly anisotropic. In terms of a distance function, the description of visual space must contain an expanding and a compressing part for all subjects.

\section{REFERENCES}

Battro, A. M., di Pierro Netto, S., \& Rozestraten, J. A. (1976). Riemannian geometries of variable curvature in visual space: Visual alleys, horopters, and triangles in big open fields. Perception, 5, 9-23.

Battro, A. M., Reggini, H. C., \& Karts, C. (1978). Perspectives in open spaces: A geometrical application of the Thouless index. Perception, 7, 583-588.

Blank, A. A. (1958), Analysis of experiments in binocular space perception. Journal of the Optical Society of America, 48, 911-925.

Blank, A. A. (1961). Curvature of binocular visual space: An experiment. Journal of the Optical Society of America, 51, 335-339.

BLANK, A. A. (1978). Metric geometry in human binocular perception: Theory and fact. In E. L. J. Leeuwenberg \& H. F. J. M. Buffart (Eds.), Formal theories of visual perception (pp. 83-102). New York: Wiley.

BLUMENFELD, W. (1913). Untersuchungen über die scheinbare Grösse in Sehräume [Studies on apparent sizes in visual space]. Zeitschrift für Psychologie, 65, 241-404.

CollewiJn, H., \& ERKelens, C. J. (1990). Binocular eye movements and the perception of depth. In E. Kowler (Ed.), Eve movements and their role in visual and cognitive processes (pp. 213-261). Amsterdam: Elsevier.

Ellis, S. R., Smith, S., Grunwald, A., \& McGreevy, M. W. (1991). Direction judgment error in computer generated displays and actual scenes. In S. R. Ellis (Ed.), Pictorial communication in virtual and real environments (Pt. IV, Chap. 34, pp. 504-526). London: Taylor \& Francis.

EsCHENBURG, J. C. (1980). Is binocular visual space constantly curved? Journal of Mathematical Biology, 9, 3-22.

Foley, J. M. (1963). Desarguesian property in visual space. Journal of the Optical Society of America, 54, 684-692.

FOLEY, J. M. (1965). Locus of perceived equidistance as a function of viewing distance. Journal of the Optical Society of America, 56, 822827.

FoLEY, J. M. (1972). The size-distance relation and intrinsic geometry of visual space: Implications for processing. Vision Research, 12. 323-332.

FoLEY, J. M. (1977), Effect of distance information and range on two indices of visually perceived distance. Perception, 6, 449-460.

Foley, J. M. (1980). Binocular distance perception. Psychological Review, 87, 411-434.

FOLEY. J. M. (1991). Binocular space perception. In D. Regan (Ed.), Vision and visual dysfunction: Binocular vision (Vol. 9, Chap. 4. pp. 75-92). London: Macmillan.

Gilinsky, A. S. (1951). Perceived size and distance in visual space. $P s y^{\prime}$ chological Review, 58, 460-482.

HaRdy, L. H., RAND, G., \& RitTler, M. C. (1951). Investigation of visual space: The Blumenfeld alleys. Archives of Ophthalmologv, 45, 53-63.

Heller, J. (1997a). On the psychophysics of binocular space perception. Journal of Mathematical Psychology, 41, 29-43.

Heller, J. (1997b). Psychophysische Invarianzen des beidäugigen Raumsehens [Psychophysical invariances in binocular space perception]. Zeitschrift für Psychologie, 205, 297-318.

HigashiYAmA, A. (1981). Variation of curvature in binocular visual 
space estimated by the triangle method. Vision Research, 21, 925933.

Higashiyama, A. (1984). Curvature of binocular visual space: A modified method of right triangle. Vision Research, 24, 1713-1718.

Hillebrand, F. (1902). Theorie der scheinbaren Grösse bei binocularem Sehen [Theory of apparent sizes in binocular vision]. Denkschriften der Wiener Akademie, Mathematisch-Naturwissenschaft Klasse, 72, 255-307.

INDow, T. (1991). A critical review of Luneburg's model with regard to global structure of visual space. Psychological Review, 98, 430-453.

INDOw, T. (1997). Hyperbolic representation of global structure of visual space. Journal of Mathematical Psychologv, 41, 89-98.

Indow, T., Inoue, E., \& Matsushima, K. (1962a). An experimental study of the Luneburg theory of binocular space perception: 1. The 3- and 4-point experiments. Japanese Psychological Research, 4, 616.

Indow. T. InOUf., E.. \& Matsushima, K. (1962b). An experimental study of the Luneburg theory of binocular space perception: 2 . The alley experiments. Japunese Psychological Research, 4, 17-24.

InDOW. T.. \& WATANaBE. T. (1984). Parallel-alleys and distance-alleys on horopter plane in the dark. Perception, 13, 165-182.

KOENDERINK, J. J., \& VAN DOORN, A. J. (1998). Exocentric pointing. In
L. R. Harris \& M. Jenkin (Eds.), Vision and action (pp. 295-313). Cambridge: Cambridge University Press.

LuKas, J. (1983). Visuelle Frontalparallelen: Ein Entscheidungsexperiment zu den Theorien von Blank, Foley und Luneburg [Visual frontoparallel horopters: A decision experiment between the theories of Blank, Foley, and Luneburg]. Zeitschrift fïr Experimentelle und Angewandte Psychologie, 4, 610-627.

LUNEBURG, R. K. (1947). Mathematical analysis of binocular vision. Princeton, NJ: Princeton University Press.

StOKER, J. J. (1969). Differential geometry. New York: Wiley.

WAGNER, M. (1985). The metric of visual space. Perception \& Psychophysics, 38, 483-495.

ZAJACZKOWSKA, A. (1956a). Experimental determination of Luneburg's constants $\sigma$ and K. Quarterly Journal of Experimental Psychology, 8 , 66-78.

ZaJaCZKOWSKA, A. (1956b). Experimental test of Luneburg's theory: Horopter and alley experiments. Journal of the Optical Society of America, 46, 514-527.

(Manuscript received December 31, 1998; revision accepted for publication December 2, 1999.) 